\title{
THE UNION LIST OF SERIALS IN ASTRONOMY LIBRARIES OF THE UK
}

\author{
A.R. Macdonald, Royal Observatory, Edinburgh
}

\section{HISTORY}

The compilation of a common listing of the serials holdings of the main astronomy libraries in the UK was proposed at the first Meeting of Astronomy Librarians of the UK held at the Institute of Astronomy in Cambridge in March 1980. The Library of the Royal Observatory in Edinburgh was at that time already engaged in a project to catalogue its serials holdings and at the same time to compile a separate, briefer, computerised listing for use in the library. Although the original idea had been that the proposed union list should record only the astronomy serials held by the participating libraries, the Royal Observatory Library offered to assume responsibility for the project as a natural development of its own list and to construct a union list that would record the complete serials holdings of all the participating libraries as it was felt that this would be of even greater benefit to these libraries.

Because the work on the project could proceed only as a background activity within the Library (the Librarian was both editor and compiler) and because software for the project was still to be developed, the project could not be started in earnest until 1984. The delay meant, however, that we were able to concentrate on the computerisation of our own list and this was completed towards the end of 1983.

Of the institutions represented at the meeting in 1980 the following have been included in the project: the Institute of Astronomy at Cambridge, Nuffield Radio Astronomy Laboratories at Jodrell Bank, the Royal Astronomical Society, the Royal Greenwich Observatory and ourselves at Edinburgh.

It is possible that we might also be able to incorporate selected holdings from libraries covering supporting subjects and from astronomical collections in other public-access libraries. We have in fact already been invited to include the astronomical holdings of such major libraries as the British Library Lending Division and the new Scottish Science Library which will open in Edinburgh in 1989.

\section{SOFTWARE}

Software for the project was written in-house originally in FORTRAN IV but was subsequently rewritten in FORTRAN 77 and a recent development has been the construction of a screen-based file management system which will offer a more efficient and user-friendly method of working with the data.

\section{FACILITIES}

We can provide at present the following types of output:

The Union List itself and: 
i.

A list of each library's own holdings (full listing or current titles only)

ii. Listing by location within each library (full listing or current titles only)

iii. Shelflists (full listing, or current titles only)

and even....

iv. Listing of serials not held by a library but held by other participating libraries. This is useful as a stockcheck.

And should in future also be able to offer:

i. Listings of observatory publications (by country $A-Z$ and alphabetically by place within each country)

ii. Listings of observatory publications alphabetically by observatory

Whether the list could be developed further $(\mathrm{e} . \mathrm{g}$. , in conjunction with the US and any other such Union list) and whether indeed it should be so developed are possible topics for discussion. However, it is a fact that we ourselves, knowing the demand that such a project makes on the resources of a busy research library, would not be able to contemplate the diversion of further effort into such a project but would nevertheless be willing to contribute data.

\section{FORMATS}

The Union list itself and the separate listing of each library's own holdings have until now been distributed to participating libraries on standard computer printout. The list continues to grow, however, and this method of publication will become more impractical. The number of entries on the file at present has reached 3400 and will exceed 4000 by the time all of the holdings have been incorporated. This already represents over 500 pages of computer printout and we are now therefore considering other means of distributing the list. Microfiche is the most obvious immediate alternative but it might also be possible to make the file available on-line over the academic networks such as JANET or even to offer it to one of the information retrieval services such as ESA-IRS or DIALOG. What exactly that would involve, however (who, for example, would provide the necessary software), has not been investigated.

\section{CONCLUSIONS}

When we originally agreed to take this project on and further proposed that its scope should be enlarged, we did so in the belief that it would be 'a good thing to do' and were, perhaps naively, not too daunted by the task. Because the project has taken so long our enthusiasm for it has at times been sorely tried, but now that the end of the project is in sight (in so far as this stage can be called the end) we are keen to continue. We believe that our initial enthusiasm for the list will prove not to have been misplaced and the community of astronomical libraries will derive some benefit from it. 